\title{
The gem-Dimethyl Effect Revisited
}

\author{
Steven M. Bachrach* \\ Department of Chemistry, Trinity University, 1 Trinity Place, San Antonio Texas 78212 \\ sbachrach@trinity.edu
}

\section{Supporting Information Available}

Table of Contents

Full citations for references 22 and $23 \ldots \ldots . . .1$

Electronic energies and ZPVEs..............2

\section{Full citations for references 22 and 23.}

(22) Frisch, M. J.; Trucks, G. W.; Schlegel, H. B.; Scuseria, G. E.; Robb, M. A.; Cheeseman, J. R.; Montgomery, J., J. A.; Vreven, T.; Kudin, K. N.; Burant, J. C.; Millam, J. M.; Iyengar, S. S.; Tomasi, J.; Barone, V.; Mennucci, B.; Cossi, M.; Scalmani, G.; Rega, N.; Petersson, G. A.; Nakatsuji, H.; Hada, M.; Ehara, M.; Toyota, K.; Fukuda, R.; Hasegawa, J.; Ishida, M.; Nakajima, T.; Honda, Y.; Kitao, O.; Nakai, H.; Klene, M.; Li, X.; Knox, J. E.; Hratchian, H. P.; Cross, J. B.; Adamo, C.; Jaramillo, J.; Gomperts, R.; Stratmann, R. E.; Yazyev, O.; Austin, A. J.; Cammi, R.; Pomelli, C.; Ochterski, J. W.; Ayala, P. Y.; Morokuma, K.; Voth, G. A.; Salvador, J.; Dannenberg, J. J.; Zakrzewski, V. G.; Dapprich, S.; Daniels, A. D.; Strain, M. C.; Farkas, O.; Malick, D. K.; Rabuck, A. D.; Raghavachari, K.; Foresman, J. B.; Ortiz, J. V.; Cui, Q.; Baboul, A. G.; Clifford, S.; Cioslowski, J.; Stefanov, B. B.; Liu, G.; Liashenko, A.; Piskorz, P.; Komaromi, I.; Martin, R. L.; Fox, D. J.; Keith, T.; Al-Laham, M. A.; C. Y. Peng, C. Y.; Nanayakkara, A.; Challacombe, M.; Gill, P. M. W.; Johnson, B.; Chen, W.; Wong, M. W.; Gonzalez, C.; Pople, J. A.; GAUSSIAN-03, rev. B.03; Gaussian, Inc.: Pittsburgh PA, 2003.

(23) Bylaska, E. J.; de Jong, W. A.; Kowalski, K.; Straatsma, T. P.; Valiev, M.; Wang, D.; Aprà, E.; Windus, T. L.; Hirata, S.; Hackler, M. T.; Zhao, Y.; Fan, P.-D.; Harrison, R. J.; Dupuis, M.; Smith, D. M. A.; Nieplocha, J.; Tipparaju, V.; Krishnan, M.; Auer, A. A.; Nooijen, M.; Brown, E.; Cisneros, G.; Fann, G. I.; Früchtl, H.; Garza, J.; Hirao, K.; Kendall, R.; Nichols, J. A.; Tsemekhman, K.;

Wolinski, K.; Anchell, J.; Bernholdt, D.; Borowski, P.; Clark, T.; Clerc, D.; Dachsel, H.; Deegan, M.; Dyall, K.; Elwood, D.; Glendening, E.; Gutowski, M.; Hess, A.; Jaffe, J.; Johnson, B.; Ju, J.; Kobayashi, R.; Kutteh, R.; Lin, Z.; Littlefield, R.; Long, X.; Meng, B.; Nakajima, T.; Niu, S.; Pollack, L.; Rosing, M.; Sandrone, G.; Stave, M.; Taylor, H.; Thomas, G.; van Lenthe, J.; Wong, A.; Zhang, Z.; NWCHEM, ver. 5.0; Pacific Northwest National Laboratory: Richland, WA, 2006. 
Electronic energies (hartrees) and zero-point vibrational energies (kcal mol ${ }^{-1}$ )

\begin{tabular}{|c|c|c|c|}
\hline Method & Cyclobutane $\mathbf{1}$ & Methylcyclobutane $\mathbf{2}$ & 1,1-Dimethylcyclobutane $\mathbf{2}$ \\
\hline G2MP2 (0 K) & -156.855422 & -196.08352 & -235.315048 \\
\hline G3MP2B3 (0 K) & -156.901787 & -196.141364 & -235.384581 \\
\hline PBE1PBE $^{a}$ & -157.065268 & -196.344072 & -235.624639 \\
\hline ZPVE & 69.6342 & 87.20299 & 104.57437 \\
\hline MO5-2X ${ }^{b}$ & -157.221051 & -196.540823 & -235.863133 \\
\hline ZPVE & 70.72 & 87.838 & 105.526 \\
\hline \multirow[t]{2}{*}{$\operatorname{CCSD}(\mathrm{T})^{c}$} & -156.834082 & -196.057406 & -235.284213 \\
\hline & Methane & Ethane & Propane \\
\hline G2MP2 (0 K) & -40.409664 & -79.62893 & -118.853077 \\
\hline G3MP2B3 (0 K) & -40.424364 & -79.65549 & -118.891369 \\
\hline PBE1PBE $^{a}$ & -40.4737706 & -79.7483979 & -119.0254303 \\
\hline ZPVE & 28.08067 & 46.86165 & 64.88917 \\
\hline MO5-2X ${ }^{b}$ & -40.5125003 & -79.8265369 & -119.1437952 \\
\hline ZPVE & 28.314 & 47.822 & 66.296 \\
\hline \multirow{2}{*}{$\operatorname{CCSD}(\mathrm{T})^{c}$} & -40.4151295 & -79.6313305 & -118.8514915 \\
\hline & 2-methylpropane & 2,2-dimethylpropane & \\
\hline G2MP2 (0 K) & -158.080807 & -197.310521 & \\
\hline G3MP2B3 (0 K) & -158.130718 & -197.371419 & \\
\hline $\mathrm{PBE}_{\mathrm{PBE}}^{a}$ & -158.30369 & -197.582037 & \\
\hline ZPVE & 82.44357 & 100.34007 & \\
\hline MO5-2X ${ }^{b}$ & -158.463532 & -197.784899 & \\
\hline ZPVE & 84.258 & 102.536 & \\
\hline \multirow[t]{2}{*}{$\operatorname{CCSD}(\mathrm{T})^{c}$} & -158.07458 & -197.299514 & \\
\hline & Cyclopropane & Methylcyclopropane & 1,1-dimethylcyclopropane \\
\hline G2MP2 (0 K) & -117.628845 & -156.856539 & -196.086604 \\
\hline \multirow[t]{2}{*}{ G3MP2B3 (0 K) } & -117.663728 & -156.903088 & -196.144778 \\
\hline & Cyclopentane & Methylcyclopentane & 1,1-dimethylcyclopentane \\
\hline G2MP2 (0 K) & -196.110194 & -235.338756 & -274.56842 \\
\hline \multirow[t]{2}{*}{ G3MP2B3 $(0 \mathrm{~K})$} & -196.168926 & -235.408896 & -274.650262 \\
\hline & Pentane & & \\
\hline G2MP2 (0 K) & -197.302235 & & \\
\hline G3MP2B3 (0 K) & -197.363828 & & \\
\hline
\end{tabular}

${ }^{a} \mathrm{PBE} 1 \mathrm{PBE} / 6-311+\mathrm{G}(2 \mathrm{df}, 2 \mathrm{pd})$.

${ }^{b}$ MP5-2X/6-311+G(d,p).

${ }^{c} \operatorname{CCSD}(\mathrm{T}) / 6-31+\mathrm{G}(2 \mathrm{~d}, \mathrm{p}) / / \mathrm{PBE} 1 \mathrm{PBE} / 6-311+\mathrm{G}(2 \mathrm{df}, 2 \mathrm{dp})$. 